\title{
EL LENGUAJE CODIFICADO EN EL CHAT, CORREO ELECTRÓNICO Y MENSAJES DE TEXTOS (SMS) Y SU RELACIÓN CON LA COMPRENSIÓN DE LECTURA Y PRODUCCIÓN DE TEXTO EN ADOLESCENTES DEL NIVEL SECUNDARIO DE LIMA
}

\section{Héctor Hugo Sánchez Carlessi}

\section{Resumen}

Estudio de carácter descriptivo y comparativo orientado a identificar los niveles de comprensión de lectura y de producción de texto en dos muestras diferenciadas, de acuerdo al mayor o menor nivel de dominio o conocimiento de la terminología o lenguaje que se emplea a través del chat, correo electrónico, o mediante los mensajes cortos de texto (SMS).

Se seleccionaron de manera intencionada muestras de estudiantes del quinto año de secundaria de instituciones educativas particulares y estatales del ámbito de Lima Metropolitana y el Callao, haciendo un total de 616 estudiantes, a los cuales se les aplicó un cuestionario sobre conocimiento del vocabulario chat, correo electrónico y SMS, una prueba de comprensión de lectura y una prueba para evaluar la producción de un texto.

Los resultados permiten afirmar que los alumnos que tienen un mayor manejo o dominio del vocabulario que se emplea en el chat, correo electrónico y SMS presentan mayores niveles de comprensión de lectura y mayores niveles en la producción de texto que los alumnos que tienen menor dominio de dicho vocabulario.

Palabras Clave: Lenguaje codificado, comprensión de lectura, producción de textos. 


\section{Abstract}

It is a descriptive comparative research in order to know the reading comprehension and writing skills levels in two groups of participants with greater or lesser terminology or language mastering or knowledge related to chat, electronic mail, or short messages sentences (SMS).

The participants were intentionally chosen considering fifth grade High School students from private and public schools of Lima and Callao cities making a total of 616 students, to whom it was applied a questionnaire on knowledge of chat vocabulary, electronic mail and SMS, a reading comprehension test and another test on writing ability.

The results shows that the students with greater vocabulary command or mastering used on chat, electronic mail and SMS shows greater reading comprehension and writing ability levels than their counterparts with lesser knowledge about such vocabulary.

Key words: Coded language, reading comprehension, text production.

\section{INTRODUCCIÓN}

En plena era de la globalización y del desarrollo de la informática, los adolescentes y jóvenes de nuestro país y del mundo en general, vienen empleando con mayor frecuencia tanto el internet como el teléfono móvil en la comunicación con sus pares o a través de sus redes sociales (vía twitter, facebook, blogs u otros). Los jóvenes cotidianamente manipulan estas herramientas informáticas con las que están muy familiarizados, llámense: $\operatorname{SMS}(1)$, chat(2), twitter(3), e-mail(4), Facebook(5), blogs(6), etc. Con ellas mantienen comunicación, producen textos y desarrollan nuevos modos y prácticas de escritura.

Se puede apreciar en esta comunicación escrita el empleo de muchas contracciones en el lenguaje, empleo de íconos, emoticones, abreviaturas, símbolos gráficos, etc, que aparente- mente (para el que no conoce esto), pueden llegar a desfigurar el significado real de lo que se quiere transmitir.

En el estudio realizado por Sánchez (2011), se encontró que los estudiantes universitarios conocían 138 términos presentados en una lista de cotejos, y agregaron 48 más a ellos, es decir, un total de 186 términos.

Hay diversas opiniones respecto del impacto que causa el empleo de estas formas de lenguaje en la gramática de una lengua, e inclusive sobre la comprensión de los significados. Al decir de Rueda J. (2009), la influencia del cambio del código lingüístico puede llegar a ejercer sobre los usuarios debe ser entendida como una involución educativa $\mathrm{y}$ un obstáculo para reconocer los patrones normativos de la lengua española. En otras palabras, el uso de contracciones y jerga electrónica no representa un ejercicio inductor de una mayor competencia 
oral y/o escrita de los jóvenes, sino más bien la entorpece.

Estas comunicaciones alteran la estructura del lenguaje formal, y pueden derivar a la larga en una alteración de los significados, y por ende, en la estructura del pensamiento, (Rueda, Juan 2009)

Se puede afirmar también que estas formas de lenguaje que emplean los adolescentes y jóvenes a través de estos medios, pueden estar trastocando su comprensión de lectura y su habilidad para componer textos escritos, comprometiendo así aspectos psicolingüísticos desde el nivel secundario.

\section{ANTECEDENTES}

E1 estudio más reflexivo sobre el impacto social y cognitivo de los mensajes de textos (SMS) es el de Alonso y Perea (2008). Igualmente, Galera J. (2010) ubica un nuevo escenario de socialización del lenguaje del adolescente: "El Chat". Barrera L (2009) presenta al Chat como una nueva función metalingüística, y Avendaño V. (2007) presenta al Chat $\mathrm{y}$ a los SMS como un nuevo género discursivo que amerita un estudio psicolingüístico.

Respecto a estudios empíricos, citamos a Lunsford Andrea (2010), de la Universidad de Stanford, que realizó una investigación por cinco años (2001-2006). En ella se analizaron 15.000 escritos de alumnos universitarios, incluyendo ensayos académicos, trabajos en clase, correos electrónicos, publicaciones en blogs, actualizaciones en espacios sociales y sesiones de chat. Haven (2009)

Este estudio arrojó sorprendentes resultados que ponen en cuestión lo que por lo general piensa el común de la gente. Para los jóvenes, para quienes es algo común pasar mucho más tiempo escribiendo, debido sobre todo a que la comunicación en las redes sociales (Facebook, Twitter, otras), es en gran medida textual. Contrariamente a lo que muchos creen -según Lunsford-, los jóvenes saben reconocer el contexto comunicacional y utilizan lenguajes y estilos diferentes según corresponda en la ocasión: son capaces de adaptarse a las condiciones de producción de cada tipo textual, es decir, de cambiar de registro.

El presente estudio tiene como principal antecedente el proyecto: "El empleo del lenguaje codificado en el chat y correo electrónico y su relación con la comprensión de lectura y producción de texto en adolescentes y jóvenes de Lima", proyecto que se realizó en el año 2011 y que comprendió una muestra de estudiantes universitarios que cursaban el primer año de estudios en dos universidades de Lima, una particular y otra estatal. Se llegó a los siguientes resultados:

$1^{\circ}$ Los alumnos universitarios, tanto de universidad estatal como particular, vienen haciendo uso del vocabulario chat y SMS de una manera que cada vez más va en aumento o incremento.

$2^{\circ}$ En los estudiantes de la universidad particular, los alumnos que tie- 
nen mayor conocimiento del vocabulario chat y SMS, presentan menor nivel en la comprensión de lectura crítica que los alumnos que tienen menor conocimiento de dicho vocabulario.

$3^{\circ}$ En los estudiantes de la universidad estatal, los alumnos que tienen mayor conocimiento del vocabulario chat y SMS, presentan mayor nivel en la comprensión de lectura crítica que los alumnos que tiene menor conocimiento de dicho vocabulario.

$4^{\circ}$ En ambos grupos de estudio de alumnos de una universidad particular y una estatal, no se ha encontrado relaciones entre el mayor conocimiento del vocabulario chat y SMS y los indicadores de producción de textos escritos.

\section{MARCO CONCEPTUAL}

El marco conceptual del estudio recoge la totalidad de planteamientos teóricos formulados en el estudio del 2011, cuyo contenido aborda aspectos vinculados con el lenguaje y sus principales características; las funciones del lenguaje incidiendo en la función comunicativa y expresiva. Así mismo, considera aspectos vinculados con la comprensión de la lectura y la producción de textos en el nivel de educación secundaria.

\section{El lenguaje y la jerga}

La Jerga es el nombre que recibe una variedad del habla diferenciada de la lengua estándar, la cual es incluso a veces incomprensible para los hablantes de ésta. Es usada con frecuencia por distintos grupos sociales con intenciones de ocultar el verdadero significado de sus palabras. Normalmente, los términos usados en la jerga de grupos específicos son temporales (excepto las jergas profesionales), perdiéndose el uso poco tiempo después de ser adoptados.

\section{El lenguaje a través del Chat y los SMS}

Muchas personas, especialmente adolescentes y jóvenes, se comunican por escrito mediante el correo electrónico y de manera directa e inmediata a través del chat, para lo cual se ha creado el término chatear que es una conversación escrita en tiempo real. Así mismo, mediante los teléfonos móviles se comunican en forma escrita mediante mensajes cortos de texto. (SMS). Lo que habría que preguntarse es respecto al tipo de lenguaje que emplean, pues muchas veces son abreviaturas, otras veces contracciones, y en otras emplean gráficos que suponen emociones.

\section{La comprensión de la lectura}

La comprensión es una actividad racional que supone el descubrimiento de lo característico o esencial en los objetos y fenómenos de la realidad. En tal sentido, una condición indispensable para el éxito de la comprensión es la riqueza y variedad de conocimientos que se va logrando.

\section{La Capacidad de Producción de Texto}

La producción de textos constituye una capacidad psicológica y lingüística que le permite a la persona elaborar $\mathrm{y}$ organizar significativa y adecuadamente un texto escrito. 


\section{Definición de Texto}

De manera general un texto es una unidad lingüística comunicativa que concreta una actividad verbal con carácter social en que la intención del hablante produce un cierre semánticocomunicativo, de modo que el texto es autónomo. El texto presenta una estructura orgánica, es decir, está constituido por partes relacionadas de tal modo que no pueda eliminarse una sin destruir la totalidad. De forma general podemos considerar como partes constitutivas del texto: el enunciado y el párrafo.

El párrafo es una unidad de significado, porque desarrolla una idea completa y distinta de las de los demás párrafos. Pero además, es también una unidad visual porque los párrafos se separan entre sí mediante los signos de puntuación.

\section{La Producción de Textos}

Escribir constituye una competencia fundamentalmente comunicativa que involucra procesos de planificación, teatralización y revisión del texto. Producir textos implica la construcción de significados para expresar diversos propósitos mediante variados tipos de textos. También incluye estrategias para reflexionar sobre lo producido, de tal manera que el niño sea consciente de sus propios procesos de aprendizaje.

\section{OBJETIVOS ESPECÍFICOS}

1) Identificar los niveles de conocimiento del lenguaje codificado que se emplea en el chat, correo electrónico y SMS, en adolescentes del nivel secundario de Instituciones Educativas Particular y Estatal de Lima Metropolitana.

2) Establecer la relación entre el mayor conocimiento del lenguaje codificado del chat y correo electrónico y SMS con el nivel de comprensión de lectura en adolescentes del nivel secundario de Lima.

3) Establecer la relación entre el conocimiento del lenguaje codificado del chat y correo electrónico y SMS con el nivel de producción de texto escrito en adolescentes del nivel secundario de Lima.

\section{MÉTODO HIPÓTESIS}

\section{Hipótesis Central}

El mayor conocimiento del lenguaje codificado que emplean en el chat, correo electrónico y SMS, está asociado a los niveles de comprensión de lectura y a los niveles de producción de textos escritos en adolescentes del nivel secundario de Lima.

\section{Hipótesis específicas}

H1 Los adolescentes de Lima metropolitana del nivel de educación secundaria que tienen mayor conocimiento del lenguaje del chat, correo electrónico y empleo del SMS presentan bajos niveles de comprensión de lectura en comparación con aquellos que tienen un menor conocimiento y dominio del lenguaje del chat, empleando un lenguaje más formal.

H2 Los adolescentes de Lima Metropolitana del nivel secundario que tienen mayor conocimiento del lengua- 
je del chat, correo electrónico y SMS presentan bajos niveles de producción de texto escrito que aquellos que tienen un menor conocimiento y dominio y emplean un lenguaje más formal,

\section{Método de investigación}

El estudio se caracteriza por ser de carácter exploratorio, descriptivo y comparativo. Está orientado al establecimiento de relaciones entre la variable conocimiento de vocabulario o terminología del chat, correo electrónico y SMS con las variables niveles de comprensión de lectura y producción de textos en adolescentes secundarios.

\section{Diseño de investigación}

El estudio es descriptivo (Sánchez, 2006) o no experimental (Hernández, 2010) y el diseño especifico es comparativo (Sánchez, 2006)

\section{Muestra de estudio}

Estuvo conformada por estudiantes del quinto año de educación secundaria. Se partió de dos muestras iniciales de mayor número, extraídas de Instituciones Educativas Particular y Estatal y, a partir de estas, se seleccionaron y conformaron 4 grupos muestrales específicos de manera intencionada: 2 grupos de I.E. estatales y 2 grupos de I.E. particulares, con las siguientes características

Grupo A1. Estudiantes de quinto de secundaria de I.E. Particular, Grupo Alto (con mayor conocimiento del vocabulario chat y SMS).

Grupo A2. Estudiantes del quinto de secundaria I.E. Particular, Grupo Bajo (con menor conocimiento del vocabulario chat y SMS).
Grupo B1. Estudiantes de quinto de secundaria de I.E. Estatal, Grupo Alto (con mayor conocimiento del vocabulario chat y SMS).

Grupo B2. Estudiante de quinto de secundaria de I.E. Estatal, Grupo Bajo (con menor conocimiento del vocabulario chat y SMS).

\section{Instrumentos de recogida de datos}

Fueron conformados por:

1.- Un cuestionario a manera de lista de cotejo sobre conocimiento de términos o palabras empleadas en el chat, correo electrónico y SMS.

2.- Un prueba de comprensión de lectura conformada por dos párrafos de lectura de 7 reactivos de alternativa múltiple cada una. El puntaje máximo a lograr fue de 14 y el mínimo de cero (0).

3.- Una prueba de producción de texto consistente en la elaboración de un ensayo que tenía que redactar cada alumno sobre la amistad.

Los textos redactados fueron evaluados considerando los siguientes indicadores: Cantidad de palabras, número de párrafos, coherencia entre párrafos, número de errores ortográficos, sintaxis, número de sustantivos empleados y originalidad.

\section{RESULTADOS OBTENIDOS}

\section{COMPOSICIÓN DE LA MUESTRA INICIAL TOTAL}

Las muestras de estudio estuvieron conformadas inicialmente por 317 alumnos del quinto año de secundaria de Instituciones Educativas Particulares y 299 alumnos del quinto año de 
secundaria de Instituciones Educativas Estatales. La totalidad de la muestra inicial estuvo conformada por 616 alumnos. Las I.E. Particulares fueron: Alcides Vigo (ubicado en Surco), Liceo Naval Montero (ubicado en el Cercado de Lima), Santa Isabel (ubicado en Breña), Junior César de los Ríos (ubi- cado en el Callao), San Patricio (ubicado en Miraflores) y San Pio X (ubicado en los Olivos). Las I.E. Estatales fueron: Dora Mayer (ubicado en el Ca1lao), Juana Alarco de Dammert (ubicado en Surco), Rosa de Santa María (ubicado en Breña), Hipólito Unanue (ubicado en el cercado de Lima)

TABLA $\mathbf{N}^{\circ} 01$. Composición de la muestra inicial

\begin{tabular}{lclc}
\hline Nombre de la I.E. Particular & N & Nombre de la I.E. Estatal & N \\
\hline Alcides Vigo & 60 & Dora Mayer & 59 \\
Liceo Naval Montero & 78 & Juana Alarco de Dammert & 57 \\
Santa Isabel & 44 & Rosa Santa Maria & 47 \\
Junior Cesar de los Rios & 64 & Hipólito Unanue & 62 \\
San Patricio (Miraflores) & 24 & Jorge Chávez (Surco) & 74 \\
San Pio X (los Olivos) & 47 & & $\mathbf{2 9 9}$ \\
\hline TOTAL & $\mathbf{3 1 7}$ & TOTAL &
\end{tabular}

\section{COMPOSICIÓN DE LOS GRUPOS MUESTRALES DEFINITIVOS}

Tal como se observa en la tabla 02, las muestras se conformaron a partir de la selección del $27 \%$ superior y del $27 \%$ inferior del total de alumnos de las instituciones educativas Particular y Estatal que respondieron respecto del mayor o menor conocimiento del vocabulario empleado a través del Chat y del SMS. Para el caso de los alumnos de I. E. Particular, se consideraron dos grupos conformados por 86 alumnos cada grupo.

El grupo superior A1 (Grupo Alto) comprendía a los alumnos que conocían un mayor número de términos o vocabulario empleados en el chat y SMS; en tanto que el grupo inferior A2 (Grupo Bajo) comprendía a los alumnos que tenían un menor conocimiento de la terminología o vocabulario empleados en el Chat y SMS. De igual manera, se procedió con los alumnos de I.E. Estatal, definiendo a 82 alumnos con alto conocimiento de la terminología chat y SMS B1 (Grupo Alto) y 83 a alumnos con bajo conocimiento de dicha terminología B2 (Grupo Bajo).

TABLA N ${ }^{\circ}$ 02. Composición de la muestra definitiva

\begin{tabular}{l|l|l}
\hline \multicolumn{1}{c}{ TIPO DE I.E. } & \multicolumn{1}{l}{$\begin{array}{l}\text { GRUPO } \\
\text { ALTO }\end{array}$} & \multicolumn{1}{l}{$\begin{array}{l}\text { GRUPO B } \\
\text { AJO }\end{array}$} \\
\hline I. E. Particular & A1 86 & A2 86 \\
\hline I. E. Estatal & B1 82 & B2 82 \\
\hline Total & 168 & 168 \\
\hline
\end{tabular}

3. RESULTADOS DE LA APLICACIÓN DEL CUESTIONARIO DE VOCABULARIO CHAT Y SMS EN LA MUESTRA TOTAL INICIAL

En la Tabla 03, se observa que los alumnos I.E. Particulares obtienen ligeramente un mayor conocimiento 
del vocabulario chat y SMS que los alumnos de I.E. Estatales, aunque al parecer las diferencias no son significativas (Medias de 75.48 y 71.90 respectivamente). Al comparar con los resultados de los universitarios en el estudio anterior (Sánchez 2011), se observa que los resultados son mayores que los obtenidos por los estudiantes secundarios, lo que se explicaría por la mayor experiencia del universitario y las mayores posibilidades de hacer uso de los medios virtuales de comunicación. En ambos casos, los alumnos de I.E. Particulares tienen mayor conocimiento de vocabulario chat que los alumnos de centros estatales.

TABLA 03.- Valores estadísticos obtenidos en el cuestionario sobre conocimiento de vocabulario chat y SMS.

\begin{tabular}{ccc}
\hline & $\begin{array}{c}\text { I.E. } \\
\text { Particular }\end{array}$ & I.E. Estatal \\
\hline Media & 75.48 & 71.90 \\
\hline Mediana & 77.00 & 72.00 \\
\hline Desv. típ. & 35.324 & 32.257 \\
\hline Mínimo & 1 & 0 \\
\hline Máximo & 153 & 147 \\
\hline Rango & 152 & 147 \\
\hline
\end{tabular}

\section{RESULTADOS OBTENIDOS EN LA PRUEBA DE COMPRENSIÓN DE LECTURA EN LA MUESTRA TOTAL}

Para tener una apreciación general del nivel de comprensión de lectura considerando tipos de centro educativo, en la Tabla $\mathrm{N}^{\circ} 04$ se presentan las medidas estadisticas de ambas muestras generales. Sobre un puntaje máximo de 14 y mínimo de 0 , se obser- va que los alumnos de I.E. Particular obtienen ligeramente un mayor rendimiento promedio que los alumnos de I.E. Estatales. Esta diferencia es mayor cuando se considera a la mediana como medida de tendencia central (10.00 y 8.00 respectivamente).

\section{TABLA 4.- Valores estadísticos} obtenidos en la prueba de comprensión de lectura

\begin{tabular}{|c|c|c|}
\hline & $\begin{array}{c}\text { I.E. } \\
\text { Particular }\end{array}$ & I.E. Estatal \\
\hline Media & 9.10 & 8.07 \\
\hline Mediana & 10.00 & 8.00 \\
\hline Desv. típ. & 2.61 & 2.6 \\
\hline Mínimo & 1 & 1 \\
\hline Máximo & 14 & 14 \\
\hline Rango & 13 & 13 \\
\hline
\end{tabular}

5. RESULTADOS OBTENIDOS CONSIDERANDO LAS MUESTRAS DEFINITIVAS (GRUPOS: ALTO Y BAJO) DE INSTITUCIÓN EDUCATIVA PARTICULAR

\subsection{CONOCIMIENTO DE TERMINO- LOGÍA CHAT Y SMS}

En relación al conocimiento del vocabulario o terminología chat y SMS en centros particulares, la Tabla $\mathrm{N}^{\circ}$ 05 presenta resultados estadísticos totalmente diferentes entre los grupos Alto y Bajo. Al aplicarse la prueba t de significación estadística, se obtiene un valor de 41.94, que es altamente significativo como para afirmar que el grupo alto es representativo de un grupo muestral que tiene un mayor conocimiento de la terminología Chat y SMS. 
El lenguaje codificado en el Chat, Correo electrónico y mensajes de textos (SMS)

\begin{tabular}{|c|c|c|}
\hline Estadist. & Grupo Alto & Grupo Bajo \\
\hline \multicolumn{3}{|l|}{ Media } \\
\hline Mediana & 116 & 28 \\
\hline Desv. típ. & 11.41 & 15.79 \\
\hline Minimo & 102 & 1 \\
\hline Máximo & 153 & 53 \\
\hline Rango & 51 & 52 \\
\hline $\mathrm{T}$ & 41.94 & \\
\hline Diferencia & 88.15 & \\
\hline Significat & 0 & \\
\hline
\end{tabular}

\subsection{COMPRENSIÓN DE LECTURA}

En cuanto a comprensión de lectura, la tabla 06 presenta al grupo alto con un mayor puntaje promedio que el grupo bajo (9.50 y 8.58 respectivamente). Al aplicarse la prueba de significación, se obtiene una $\mathrm{t}$ de 2.22 , que es significativa al $0.03 \%$ de probabilidad. Se puede afirmar que el grupo que tiene un mayor conocimiento del vocabulario Chat y SMS también tiene un mayor nivel de comprensión de lectura.

\section{TABLA 6. Valores estadísticos} obtenidos en comprensión de lectura considerando grupos extremos en I.E. Particular

\begin{tabular}{lcc}
\hline Estadist. & Grupo Alto & Grupo Bajo \\
\hline Media & 9.50 & 8.58 \\
\hline Mediana & 10.00 & 10.00 \\
\hline Desv. típ. & 2.44 & 2.72 \\
\hline Mínimo & 1 & 1 \\
\hline Máximo & 13 & 13 \\
\hline Rango & 12 & 12 \\
\hline
\end{tabular}

\begin{tabular}{ll}
\hline $\mathrm{t}$ & 2.22 \\
\hline Diferencia & 0.92 \\
\hline Significa & 0.03 \\
\hline
\end{tabular}

\subsection{RESULTADOS EN PRODUCCIÓN DE TEXTO.}

Cada alumno debía de elaborar un texto sobre la amistad. Se consideró como indicadores cuantitativos de producción del texto los siguientes: número de palabras escritas por el alumno, número de párrafos que lograba escribir, coherencia entre párrafos, número de errores ortográficos, sintaxis de las oraciones, número de sustantivos empleados $\mathrm{y}$ palabras originales.

\subsubsection{NÚMERO DE PALABRAS.}

El grupo alto obtiene un mayor puntaje promedio que el grupo bajo. Al aplicarse la prueba de significación, la t obtenida es de 3.80 , que es altamente significativa, por lo cual se puede afirmar que los alumnos que tienen mayor conocimiento del vocabulario Chat y SMS emplean mayor número de palabras cuando redactan un texto en comparación con el grupo bajo.

TABLA 07 Valores estadísticos obtenidos en números de palabras considerando grupos extremos I.E. Particular

\begin{tabular}{lcc}
\hline & Grupo Alto & Grupo Bajo \\
\hline Media & 121.69 & 82.52 \\
\hline Mediana & 113.00 & 67.00 \\
\hline Desv. tip. & 70.701 & 72.103 \\
\hline $\mathrm{T}$ & 3,80 & \\
\hline
\end{tabular}




\begin{tabular}{lc} 
Dif medias & 39.16 \\
Significativ. & 0.00 \\
\hline
\end{tabular}

\subsubsection{NÚMERO DE PÁRRAFOS}

Los alumnos del grupo alto elaboran un mayor número de párrafos que el grupo bajo. La prueba de significación de las diferencias nos da una $t$ de 2.60, que es altamente significativa. Los alumnos de I. E. Particulares que tienen un mayor conocimiento de la terminología Chat y SMS, organizan un mayor número de párrafos en la producción de un texto.

TABLA 08. Valores estadisticos obtenidos en números de párrafos considerando grupos extremos

\section{I.E. Particular}

\begin{tabular}{lcc}
\hline & Grupo Alto & Grupo Bajo \\
\hline Media & 2.58 & 2.03 \\
\hline Mediana & 3.00 & 2.00 \\
\hline Desv. tip. & 1.393 & 1.359 \\
\hline $\mathrm{T}$ & 2.60 & \\
Dif. Medias & 0.55 & \\
Significat & 0.01 & \\
\hline
\end{tabular}

\subsubsection{COHERENCIA ENTRE PÁ- RRAFOS}

La comparación de las diferencias no muestran mayor significación, por lo cual se puede afirmar que la coherencia entre párrafos no es indicador para diferenciar los grupos altos y bajos.
TABLA 09 Valores estadisticos

obtenidos en coherencia

considerando grupos extremos

I.E. Particular

\begin{tabular}{lcc}
\hline Estadistic & Grupo Alto & Grupo Bajo \\
\hline Media & 1.99 & 1.80 \\
\hline Mediana & 2.00 & 2.00 \\
\hline Desv. tip. & .775 & .733 \\
\hline $\mathrm{t}$ & 1.62 & \\
Dif. Medias & 1.86 & \\
Significativ. & & \\
\hline
\end{tabular}

\subsubsection{ERRORES ORTOGRÁFICOS}

No hay mayores diferencias entre ambos grupos. Sin embargo, se observa que el grupo bajo tiende a tener un mayor número de errores ortográficos que el grupo alto.

TABLA 10.- Valores estadísticos obtenidos en números de errores ortográficos considerando grupos extremos I.E. Particular

\begin{tabular}{lcc}
\hline & Grupo Alto & Grupo Bajo \\
\hline Media & .97 & 1.12 \\
\hline Mediana & .00 & .00 \\
\hline Desv. tip. & 1.367 & 2.150 \\
\hline $\mathrm{t}$ & 0.55 & \\
Dif. Medias & 0.15 & \\
Significativ & 0.58 & \\
\hline
\end{tabular}

\subsubsection{SINTAXIS}

Se consideró como elemento importante mantener la estructura de las palabras en la redacción 
de la oración. En la tabla 11 se encuentra que no existen diferencias significativas entre ambos grupos; aunque el grupo alto tiende a tener una mayor sintaxis que el grupo bajo.

TABLA 11.- Valores estadísticos obtenidos en sintaxis considerando grupos extremos I.E. Particular

\begin{tabular}{lcc}
\hline & Grupo Alto & Grupo Bajo \\
\hline Media & 2.10 & 1.92 \\
\hline Mediana & 2.00 & 2.00 \\
\hline Desv. típ & .826 & .834 \\
\hline $\mathrm{T}$ & 1.47 & \\
Dif. Medias & 0.19 & \\
Significativ & 0.14 & \\
\hline
\end{tabular}

\subsubsection{SUSTANTIVOS}

En relación al empleo de sustantivos en la tabla 12, se observa que el Grupo Alto tiende a presentar más sustantivos que el Grupo Bajo. La prueba de significación entre ambos grupos arroja una $\mathrm{t}$ de 3.03 que corrobora la afirmación.

TABLA 12.- Valores estadisticos obtenidos en empleo de sustantivos considerando grupos extremos I.E. Particular

\begin{tabular}{lcc}
\hline & Grupo Alto & Grupo Bajo \\
\hline Media & 13.72 & 10.26 \\
\hline Mediana & 13.00 & 9.00 \\
\hline Desv. tip. & 7.475 & 7.501 \\
\hline $\mathrm{t}$ & 3.03 & \\
Dif. Medias & 3.46 & \\
Significativ & 0.003 & \\
\hline
\end{tabular}

\subsubsection{ORIGINALIDAD}

De acuerdo a los resultados de la tabla 13, el indicador originalidad no es significativo para ubicarlo como característico dentro de uno de los grupos de estudio. Sin embargo, la tendencia es que el grupo alto muestre textos más originales que el grupo bajo.

TABLA 13 Valores estadisticos obtenidos en originalidad considerando grupos extremos I.E. Particular

\begin{tabular}{lcc}
\hline Estadístico & Grupo Alto & Grupo Bajo \\
\hline Media & 1.13 & 1.08 \\
\hline Mediana & 1.00 & 1.00 \\
\hline Desv. típ. & .480 & .466 \\
\hline $\mathrm{t}$ & 0.64 & \\
\hline Dif. Medias & 0.05 & \\
\hline Significativ & 0.52 & \\
\hline
\end{tabular}

6. RESULTADOS OBTENIDOS CONSIDERANDO LAS MUESTRAS DEFINITIVAS (GRUPOS EXTREMOS), EN INSTITUCIONES EDUCATIVAS

\section{ESTATALES}

\subsection{VOCABULARIO CHAT}

La Tabla $\mathrm{N}^{\circ} 14$ muestra una tendencia central totalmente diferente entre los grupos alto y bajo. La $t$ obtenida de 38.91 nos permite afirmar que ambos grupos son claramente diferenciados, en función a la mayor o menor cantidad de vocabulario empleado a través del chat y del SMS. 
TABLA 14.- Valores estadísticos obtenidos en vocabulario chat y SMS considerando grupos extremos I.E. Estatal

\begin{tabular}{lcc} 
& I.E. Estatal & \\
\hline Estadist & Grupo Alto & Grupo Bajo \\
\hline Media & 112.74 & 32.52 \\
\hline Mediana & 113.00 & 37.00 \\
\hline Desv. típ. & 12.800 & 13.560 \\
\hline Minimo & 92 & 0 \\
\hline Máximo & 147 & 50 \\
\hline Rango & 55 & 50 \\
\hline T & 38.91 & \\
Dif, medias & 80.22 & \\
Significativ & 0.00 & \\
\hline
\end{tabular}

\subsection{COMPRENSIÓN DE LECTURA}

En la tabla 15, el grupo alto obtiene ligeramente un mayor puntaje que el grupo bajo, pero la diferencia no es estadisticamente significativa y no permite arribar a conclusiones.

TABLA 15.- Valores estadísticos obtenidos en comprensión de lectura considerando grupos extremos

\section{I.E. Estatal}

\begin{tabular}{lcc}
\hline & Grupo Alto & Grupo Bajo \\
\hline Media & 8.55 & 7.99 \\
\hline Mediana & 9.00 & 8.50 \\
\hline Desv. tip. & 2.500 & 2.960 \\
\hline Rango & 11 & 12 \\
\hline $\mathrm{t}$ & 1.31 & \\
Dif medias & 0.56 & \\
Significativ & 0.19 & \\
\hline
\end{tabular}

\subsection{PRODUCCIÓN DE TEXTO}

\subsubsection{NÚMERO DE PALABRAS}

De acuerdo con la tabla 16, el grupo alto obtiene mayores puntajes promedios que el grupo bajo; sin embar- go, de acuerdo con la t obtenidas, esta diferencia no resulta ser significativa. La tendencia es que los alumnos que emplean más la terminología chat y SMS empleen mayor número de palabras en la producción de un texto.

TABLA 16.- Valores estadisticos obtenidos en número de palabras considerando grupos extremos I.E. Estatal

\begin{tabular}{lcc}
\hline Estadist. & Grupo Alto & Grupo Bajo \\
\hline Media & 53.67 & 46.98 \\
\hline Mediana & 42.00 & 37.00 \\
\hline Desv. típ. & 41.14 & 46.020 \\
\hline T & 0.98 & \\
Dif medias & 6.69 & \\
Significativ & 0.33 & \\
\hline
\end{tabular}

\subsubsection{NÚMERO DE PÁRRAFOS}

En cuanto al número de párrafos, la tabla 17 muestra que el grupo alto presenta un mayor puntaje que el grupo bajo, sin embargo, la t obtenida no muestra mayores diferencias entre los dos grupos. La tendencia es que el grupo alto presente mayor número de párrafos.

TABLA 17.- Valores estadísticos obtenidos en número de párrafos considerando grupos extremos I.E. Estatal

\begin{tabular}{lcc}
\hline & Grupo Alto & Grupo Bajo \\
\hline Media & 1.77 & 1.45 \\
\hline Mediana & 1.00 & 1.00 \\
\hline Desv. típ. & 1.270 & 1.220 \\
\hline T & 1.63 & \\
Dif. Medias & 0.32 & \\
Significativ & 0.11 & \\
\hline
\end{tabular}




\subsubsection{COHERENCIA ENTRE PÁ- RRAFOS}

En cuanto al ordenamiento entre párrafos, según la Tabla 18, el grupo Alto obtiene mayor puntaje promedio que el grupo Bajo. Se confirma con la t de 3.56, que resulta altamente significativa. El grupo que conoce mayor terminología chat y SMS muestra mayor coherencia en los textos.

TABLA 18.- Valores estadisticos obtenidos en coherencia entre párrafos considerando grupos extremos

\section{I.E. Estatal}

\begin{tabular}{lcc}
\hline & Grupo Alto & Grupo Bajo \\
\hline Media & 1.96 & 1.45 \\
\hline Mediana & 2.00 & 1.50 \\
\hline Desv. tip. & .890 & .940 \\
\hline $\mathrm{t}$ & 3.56 & \\
Dif. Medias & 0.51 & \\
Significativ & 0.00 & \\
\hline
\end{tabular}

\subsubsection{NÚMERO DE ERRORES OR- TOGRÁFICOS}

En la tabla 19 se observa que no hay diferencias significativas entre los Grupos Alto y Bajo. Sin embargo la tendencia es que el grupo bajo presente mayor número de errores ortográficos.
TABLA 19.- Valores estadísticos obtenidos en número errores ortográficos considerando grupos extremos I.E. Estatal

\begin{tabular}{lcc}
\hline Estadist & Grupo Alto & Grupo Bajo \\
\hline Media & .89 & 1.07 \\
\hline Mediana & .00 & .00 \\
\hline Desv. típ. & 1.84 & 2.39 \\
\hline $\mathrm{t}$ & 0.54 & \\
\hline Dif. Medias & 0.18 & \\
\hline Significativ & 0.58 & \\
\hline
\end{tabular}

\subsubsection{SINTAXIS}

En la tabla 20 se observa que los alumnos del grupo Alto presentan en promedio mayor sintaxis que el Grupo Bajo, siendo estas diferencias significativas mayores al $0.05 \%$.

TABLA 20.- Valores estadísticos obtenidos en sintaxis considerando grupos extremos I.E Estatal

\begin{tabular}{lcc}
\hline & Grupo Alto & Grupo Bajo \\
\hline Media & 1.84 & 1.50 \\
\hline Mediana & 2.00 & 1.50 \\
\hline Desv. tip. & .84 & .99 \\
\hline $\mathrm{t}$ & 2.37 & \\
Dif medias & 0.34 & \\
Significativ & 0.02 & \\
\hline
\end{tabular}

\subsubsection{NÚMERO DE SUSTANTIVOS} En la tabla 21, si bien el grupo alto obtiene un mayor promedio de 
sustantivos que el grupo bajo, esta diferencia no es significativa. Pero la tendencia indica que los del grupo alto tienden a usar más sustantivos.

TABLA 21.- Valores estadísticos obtenidos en número de sustantivos considerando grupos extremos I.E. Estatal

\begin{tabular}{lcc}
\hline & Grupo Alto & Grupo Bajo \\
\hline Media & 7.21 & 5.85 \\
\hline Mediana & 7.00 & 5.50 \\
\hline Desv. típ. & 4.65 & 4.77 \\
\hline t & 1.84 & \\
Dif medias & 1.35 & \\
Significativ & 0.07 & \\
\hline
\end{tabular}

\subsubsection{ORIGINALIDAD}

En la tabla 22 se observa que el grupo Alto presenta un mayor puntaje promedio en originalidad que el Grupo Bajo. Estas diferencias resultan significativas superiores al $0.05 \%$.

\section{TABLA 22.- Valores estadisticos} obtenidos en originalidad considerando grupos extremos I.E. Estatal

\begin{tabular}{lcc}
\hline & Grupo Alto & Grupo Bajo \\
\hline Media & 1.12 & .90 \\
\hline Mediana & 1.00 & 1.00 \\
\hline Desv. tip. & .510 & .510 \\
\hline $\mathrm{t}$ & 2.76 & \\
Dif. Medias & 0.22 & \\
Significativ & 0.006 & \\
\hline
\end{tabular}

\section{DISCUSIÓN DE RESULTADOS}

\section{E1 Cuestionario Chat en muestras iniciales de I.E. Particular (IEP) y Estatal (IEE).}

Se observa que los alumnos de IEP obtienen ligeramente un mayor conocimiento de los términos o vocabulario Chat y SMS que los de IEE. De igual manera, este resultado resulta similar al resultado que se obtuvo con los alumnos del nivel universitario. Si bien ambos grupos muestrales obtienen menor conocimiento de la terminología chat que los alumnos de los primeros ciclos del nivel universitario, ello supone que el conocimiento de esta terminología va en aumento en función a la edad y al nivel de estudios.

\section{La prueba de comprensión de lectura en la muestra inicial total}

Comparando los resultados de la prueba de comprensión de lectura entre los dos tipos de Instituciones, se ha encontrado que los alumnos de IE Particulares presentan un mayor nivel de comprensión de lectura que los IE Estatal, siendo estas diferencias muy significativas estadísticamente. Estos resultados corroboran estudios anteriores, Sánchez (2009, 2011), en donde se ha encontrado que los alumnos IEP obtienen mejores niveles de comprensión de lectura que los alumnos IEE; resultados que se reflejan en las evaluaciones del Ministerio de Educación y, sobre todo, del programa PISA (2009), que nos ubica en los últimos niveles en cuanto a comprensión de la lectura.

\section{La comprensión de lectura con- siderando grupos extremos definiti- vos}

Estos resultados son semejantes a los hallados con los alumnos del 
nivel superior pero de universidades estatales, lo que no ocurre con los alumnos de una universidad particular. De estos hallazgos se puede inferir que los alumnos de quinto año de secundaria, poseedores de un mayor conocimiento del vocabulario chat y SMS, tienen un mayor nivel de comprensión de lectura que los que tienen un bajo conocimiento de esta terminología. Posiblemente, el hecho de que adquieran una nueva terminología basada en contracciones $\mathrm{y}$ emoticones los ubiquen con una mejor disposición para estar alerta y entender los mensajes que leen.

\section{La Producción de textos conside- rando grupos extremos definitivos}

Para el análisis y discusión de los resultados encontrados, se tomarán en cuenta los indicadores predeterminados.

Número de palabras escritas. En las I.E. Particulares, el grupo que tiene mayor conocimiento de la terminología chat presenta un mayor número de palabras que el grupo que tiene menor conocimiento (grupo bajo). En cuanto a los alumnos de I.E. Estatales, igualmente se observa que el grupo alto obtiene un mayor número de palabras que el grupo de menor conocimiento (grupo bajo). De los resultados se puede deducir que los alumnos que tienen mayor conocimiento de la terminología chat y SMS tienden a escribir un mayor número de palabras en un texto escrito.

Número de párrafos. Los alumnos de I.E. Particular que tienen mayor dominio de la terminología presentan un mayor número de párrafos que los que tienen menor dominio, siendo estas diferencias significativas estadísticamente. En cuanto a los alumnos de I.E. Estatales, se observa que los alumnos que tienen un mayor conocimiento de la terminología escriben un mayor número de párrafos que el grupo de menor conocimiento, aunque sin llegar a ser significativo estadísticamente. Estos resultados permiten afirmar que los alumnos que tienen un mayor dominio de esta terminología tienden a elaborar un mayor número de párrafos al escribir un texto.

Coherencia entre párrafos. En cuanto a la coherencia entre párrafos, en el grupo de I.E. P. se observa que el grupo alto obtiene mayor puntuación que el grupo de menor conocimiento, aunque sin llegar a ser significativo. En los alumnos de I.E.E. se observa que los del grupo alto o de mayor conocimiento de la terminología obtienen una mayor puntuación en comparación con los que presentan menor conocimiento, siendo esta diferencia estadísticamente significativa. Entonces, los alumnos con mayor dominio de la terminologia chat y SMS son más coherentes al escribir sus párrafos que los alumnos que tienen menor dominio.

Número de errores ortográficos. Para el caso de los alumnos de I.E.P. los que tienen menor dominio de la terminología chat y SMS presentan mayores errores ortográficos que los que tienen mayor conocimiento. 
Aunque esta diferencia no resulta significativa estadísticamente. En cuanto a los alumnos de I.E.E. se observa que el grupo de menor conocimiento presenta mayores errores ortográficos que el grupo de mayor conocimiento, aunque sin llegar a ser significativo estadísticamente. Se puede afirmar que hay una tendencia a que los alumnos que tienen un mayor conocimiento de la terminología chat y SMS presenten menos errores ortográficos que los que tienen menor conocimiento de esta terminología.

Sintaxis. Para el caso de los alumnos de I.E.P., se encuentra que los alumnos de mayor conocimiento presentan mayor puntuación en sintaxis que los que tienen menor conocimiento, aunque esta diferencia no es significativa. De igual manera ocurre con los alumnos de I.E.E.: los estudiantes de mayor conocimiento obtienen mayor puntuación en sintaxis que los alumnos que tienen menor conocimiento, siendo esta diferencia significativa estadísticamente. Se concluye que los alumnos que tienen mayor dominio o conocimiento en la terminologia chat y SMS tienen mayor sintaxis en la redacción de un texto que los alumnos que tienen menor conocimiento de esta terminología.

Sustantivos. En cuanto a la cantidad de sustantivos empleados, se observa que alumnos de I.E.P. que tienen mayor conocimiento o dominio de la terminología presentan mayor número de sustantivos que los estudiantes que tienen menor dominio, con diferencias estadísticamente significativas. En relación a los alumnos de I.E.E., igualmente se observa que los estudiantes que tienen un mayor conocimiento de la terminología chat y SMS presentan mayor número de sustantivos, diferencia no significativa estadísticamente. Se infiere que los estudiantes que tienen mayor conocimiento de la terminología chat y SMS emplean mayor número de sustantivos al redactar un texto que los alumnos que tienen menor conocimiento o dominio.

Originalidad. Para el caso de estudiantes de I.E.P., hay una leve tendencia a que los alumnos que tienen mayor conocimiento de la terminología muestren un texto original en comparación con los que no. Para el caso de los estudiantes de I.E.E. los alumnos que tienen mayor conocimiento de la terminología presentan mayor puntuación en originalidad en comparación con el grupo que tiene menor conocimiento, siendo esta diferencia estadísticamente significativa. Se puede concluir que los estudiantes secundarios que tienen mayor conocimiento en la terminologia chat y SMS tienden a ser más originales al escribir un texto que los alumnos que tienen menor conocimiento.

\section{En relación a la hipótesis especí- fica $\mathrm{H} 1$}

La hipótesis H1 afirma que "los adolescentes de Lima metropolitana del nivel de educación secundaria que tienen mayor conocimiento $y$ dominio del lenguaje del chat, correo electrónico 
y empleo del SMS presentan bajos niveles de comprensión de lectura en comparación con aquellos que tienen un menor conocimiento y dominio del lenguaje del chat, empleando un lenguaje más formal".

Los resultados encontrados refutan esta afirmación, ya que se ha encontrado que tanto para el caso de los estudiantes de centros educativos particulares como estatales, los alumnos que tienen un mayor conocimiento y dominio del lenguaje chat y SMS presentan mayores niveles de comprensión de lectura que aquellos alumnos que tienen un menor conocimiento. Estos resultados contradicen la opinión de algunos expertos (Alvarez, 2010), que consideran que el empleo de estas formas de lenguaje pueden distorsionar la forma como comprenden los estudiantes.

\section{En relación a la hipótesis especi- fica $\mathrm{H} 2$}

La hipótesis H2 afirma que "Los adolescentes de Lima Metropolitana del nivel secundario que tienen mayor conocimiento y dominio del lenguaje del chat, correo electrónico y SMS presentan más bajos niveles de producción de texto escrito que aquellos que tienen un menor conocimiento y dominio y emplean un lenguaje más formal".

Todos los resultados encontrados contradicen la hipótesis formulada, ya que, por el contrario, se ha encontrado que los alumnos del quinto de secundaria que tienen mayor dominio o conocimiento del lenguaje chat y SMS, presentan un mayor número de pala- bras escritas, mayor número de párrafos, mayor coherencia entre párrafos, menor número de errores ortográficos, mejor sintaxis, mayor número de sustantivos y textos más originales que los alumnos que tienen menos conocimiento del lenguaje chat y SMS.

\section{En relación a la hipótesis central}

La Hipótesis central afirma que "El mayor dominio conocimiento del lenguaje codificado que emplean en el chat, correo electrónico y SMS, está asociado a los niveles de comprensión de lectura y a los niveles de producción de textos escritos en adolescentes del nivel secundario de Lima".

En efecto, se ha encontrado una relación entre las variables conocimiento del lenguaje chat y SMS, con las variables niveles de comprensión de lectura y niveles de producción de textos. Sin embargo, esta relación contradice las hipótesis específicas, por lo que deberá precisarse que las relaciones específicas se dan en sentido contrario a lo que se esperaba.

\section{CONCLUSIONES}

En relación a los objetivos del estudio y las hipótesis planteadas, se llega a las siguientes conclusiones:

$1^{\circ}$ Los alumnos del quinto año de secundaria de instituciones educativas particulares tienen un mayor conocimiento de la terminología o vocabulario especial que se emplea a través del chat, y SMS que los alumnos del quinto año de instituciones educativas estatales. 
$2^{\circ}$ Los alumnos del quinto año de secundaria de instituciones educativas particulares tienen un mayor nivel de comprensión de lectura que los alumnos del quinto año de instituciones educativas estatales.

$3^{\circ}$ La distribución de las frecuencias sobre conocimiento de la terminología chat, correo electrónico y SMS en los alumnos del quinto año de secundaria sigue la curva normal de distribución, aunque al ser claramente sesgada a la derecha va en aumento progresivo.

$4^{\circ}$ Los alumnos del quinto año de secundaria que tienen un mayor nivel de información sobre el vocabulario o terminología que se emplea en el Chat, correo electrónico y SMS, presentan un mayor nivel de comprensión de lectura que los alumnos que tienen un bajo nivel de dominio en la terminología chat. $5^{\circ}$ Los alumnos del quinto año de secundaria que tiene un mayor nivel de información sobre el vocabulario o terminología que se emplea en el chat, correo electrónico y SMS, tienen un mejor desempeño en la producción de un texto que los alumnos que tienen bajo nivel de información en dicha terminología que se emplea en el chat, correo electrónico y SMS.

\section{NOTAS:}

1. SMS.- Mensajes corto de texto vía teléfono móvil.

2. Chat.- término proveniente del inglés; en español equivale a charla. Designa una comunicación escrita instantánea a través de Internet entre dos o más personas.

3. Twitter.- Pronunciado en inglés gorjear, parlotear, trinar, es un sitio web de microblogging que permite a sus usuarios enviar y leer micro-entradas de texto de una longitud máxima de 140 caracteres denominados "tweets".

4. E-mail.- Correo Electrónico

5. Facebook- Es un sitio web gratuito de redes sociales.

6. Blogs.- En español significa bitácora, es un sitio web periódicamente actualizado que recopila cronológicamente textos o artículos de uno o varios autores, apareciendo primero el más reciente, donde el autor conserva siempre la libertad de dejar publicado lo que crea pertinente. Versión reducida del término "web log". Es información que un usuario publica de forma fácil e instantánea en un sitio web. 
El lenguaje codificado en el Chat, Correo electrónico y mensajes de textos (SMS)

\section{REFERENCIAS}

GONZÁlEZ, Raúl (2006) Problemas Psicolingüisticos en el Perú. Páginas del Perú. SAC. Lima., p 203211219

SÁNCHEZ, Hugo (2011) Comunicación a través del chat, correo electrónico y mensajes de texto (SMS) ¿futuras formas de lenguaje y pensamiento? Revista Maestros y alumnos. Lima: Universidad Ricardo Palma

SÁNCHEZ, H. REYES C. (2006) Metodología y diseños en la investigación científica. Lima Visión Universitaria

SÁNCHEZ, Hugo (2011) El empleo del lenguaje codificado en el chat y correo electrónico y su relación con la comprensión de lectura y producción de texto en adolescentes y jóvenes de Lima. Lima; Universidad Ricardo Palma. (Informe final de investigación)

SÁNCHEZ, Hugo (2012) El lenguaje codificado a través del Chat y SMS en estudiantes universitarios de Lima. Revista Maestros y Alumnos. Lima: Universidad Ricardo Palma.

\section{REFERENCIAS WEB}

ALVAREZ G., Braulio R. (2010) ¿Es el lenguaje SMS un deterioro del idioma? Consultado el 08-06-2011 en la web braulioedunet.webcindario.com/sms. pdf.

DOMÍNGUEZ CUESTA, Carmela (s/a) El lenguaje de los SMS y del Chat en las aulas Consultado el 08-06-2011 en la web:

www.cuadernosdepedagogia.com/ver_pdf_free.asp?idArt $=8870$

LUNSFORD, A. (2010) La escritura y la profunda revolución en Access. http// proyec/infolit.org//st/lunsford.asp.

MAYANS Pianella, Joan (2000) Género confuso: Género Chat Consultado el 0806-2011 en la web: www.cibersociedad.net/textos/articulo.php?art=22- España

INFORME PISA 2009, OCDE, Informe español.

http:// www.mecd.gob.es/dctm/ministerio/horizontales / prensa/notas / $2010 / 20101207$-pisa2009-informe-espanol. pdf?documentId=0901e72b806ea35a

INFORME PISA es.wikipedia.org/wiki/Informe_PISA

ROCHA SILVA, Alejandra (2004) El lenguaje de los jóvenes en el chat Consultado el 15-06-2011 en la web: http://redalyc.uaemex.mx/src/inicio/ ArtPdfRed.jsp?iCve $=31601907$

RUEDA LÓPEZ, Juan Jesús. (2009) To chat or not to chat? He ahí la función metalingüística. Consultado de Edutec. Revista electrónica de Tecnología Educativa, página No 28. http://edutec.rediris.es/revelec2/revelec28/ 